\title{
Analysis of the Behavior Applied in the Dental Care of Children and Adolescents with Autism Spectrum Disorder
}

\author{
Roberto Carlos Mourao Pinho* \\ Department of Dental Surgery, Federal University of Pernambuco, USA \\ *Corresponding author: Roberto Carlos Mourao Pinho, Department of Dental Surgery, Federal University of Pernambuco, USA. \\ To Cite This Article: Roberto Carlos Mourao Pinho. Analysis of the Behavior Applied in the Dental Care of Children and Adolescents with Autism \\ Spectrum Disorder. Am J Biomed Sci \& Res. 2019 - 5(6). AJBSR.MS.ID.000976. DOI: 10.34297/AJBSR.2019.05.000976.
}

Received: 阱October 10, 2019; Published: 阱 October 29, 2019

\section{Mini Review}

Many children with special needs have poorer oral health than typically developing children and have difficulty obtaining health care [1], the feasibility of achieving oral health in children with ASD is associated with their developmental profiles, and, cognitive functioning levels and challenging behaviors. The challenge remains to apply and determine the effectiveness of behavioral and pedagogical interventions to ensure that more children who need special dental care in the population have access to dental care services $[2,3]$. It is essential that the autistic child always has adequate oral health and, therefore, prevention is necessary. So, from the moment parents arrive at the dental office with their children, the dentist should introduce this subject, showing the importance and, at the same time, different techniques for parents to be able to perform oral hygiene at home [4-6]. One of the techniques that can be used is the ABA, which consists in teaching new skills to the patient with ASD, and should be applied in stages, always respecting the patient's evolution, emphasizing, rewarding and reinforcing positive behaviors. ABA is another method used to teach new skills with ASD. It can also be used in dentistry, involving parents to teach their children how to brush their teeth. The technique should be introduced in stages, always rewarding the patient after completing the tasks properly in order to reinforce prevention against oral pathologies [7].

The forms of autistic patient psychological approaches are the same as those used in pediatric dentistry such as: tell-show-do, distraction, desensitization, voice control, positive reinforcement or reward, and modeling. However, these methods are more difficult to apply to autistic patients but should be encouraged. It is also possible to use body language, so that the professional, through their facial expressions, can convey to the child their satisfaction for good behavior or not. Depending on the degree of mental behavior, dental treatment of an autistic outpatient is feasible. Some details should be observed during the care of these patients, such as: elimination of stressful sensory stimuli, clear and objective orders and establishment of a care routine. Moving furniture in the office can trigger seizures and it is important that the patient is attended by the same professional in the same office with pre-established routine. The main emotion of the autistic is fear, so the environment must be calm; This facilitates consultations, despite being a major challenge [8]. In fact, the use of effective methods for it is possible, through a functional analysis, to identify different classes of behaviors and behavior control variables involved in the pediatric - child interaction; and also oral health conditions, treatment plan, level of fear and the degree of collaboration of children can be considered as conditions that establish standardized professional behaviors [9].

The child with ASD is extremely sensitive to external stimuli such as different noises, loud sounds and unexpected behaviors during dental treatment. Because of their tendency to adhere to routines, children may need multiple $C D$ visits to acclimate to the dental environment. In addition, the development of a method that provides dental professionals with a sequence of care for these patients is extremely important [10]. An important element of ABA is to analyze the function (purpose) of the behavior. Understanding the function of behavior is important when selecting which procedures to use to modify socially significant behaviors. Cases, problem behaviors are maintained by example. A child who fights in the dental chair of the treatment room may do so because of a (successful) story learned by escaping aversive situations or stimuli as a result of engaging in these behaviors. In such types of situations, undesirable behavior is accidentally reinforced by 
the caregiver and is more likely to recur on future visits. Another example is that a child may vomit in the presence of a dentist because in the past these responses have been reinforced with the closure of the procedure. Determining the variables that maintain patient behavior can help in developing treatment protocols that promote life-long dental treatment acceptance. Recommendations for home preparation not only provide opportunities for planning the next visit, but also allow the dentist to make recommendations to parents to help prepare the child prior to the appointment. Home preparation may include familiarization with instruments in educational settings; teaching prerequisite skills through ABA procedures at home, such as "open your mouth" or "counting teeth"; and develop picture books for families so they can help the child with ASD become familiar with the dental environment [11].

ABA-based procedures are evidence-based [12] and accepted by the American Academy of Pediatrics in the management of ASD [13]. In dentistry, the use of these procedures has the potential to improve the results of traditional behavior management procedures. By increasing the likelihood of patients who accept simple and routine dental procedures, dentists may decrease the need for more intrusive procedures such as restraints and sedation. However, incorporating behavioral principles into behavior management strategies is not without limitations in a busy dental practice. Although they can be effective, they can also be challenging for dental professionals, they can be time consuming and require professionals and training to implement properly. Although the pediatric dental curriculum includes many behavioral science procedures, the opportunity to have a knowledge and understanding of the fundamental principles of behavior is limited. A caregiver can benefit from having a thorough understanding of ABA principles so that he or she can modify procedures for managing a patient with ASD [13]

\section{References}

1. Cermak SA, Stein LI, Marian D, Dawson ME, Lane CJ (2015) Sensory Adapted Dental Environments to Enhance Oral Care for Children with Autism Spectrum Disorders: A Randomized Controlled Pilot Study. J Autism Dev Disord 45(9): 2876-2888.

2. Yanlin R, Cynthia D, Nigel KYY (2018) Oral Health Behaviours of Preschool Children with Autism Spectrum Disorders and Their Barriers to Dental Care. J Autism Dev Disord 49(2): 453-459.

3. Du RY, Yiu CCY, Wong VCN, Mcgrath CP (2015) Autism Developmental Profiles and Cooperation with Oral Health Screening. J Autism Dev Disord 45(9): 2758-2763.

4. Marra P (2007) Great River University Difficulties Encountered by Those Responsible for Maintaining Oral Health in People with Special Needs. Grande Rio University, USA.

5. (2014) Assisi C Specialized Care. Rev bras odontol, Rio de Janeiro p. 71.

6. Sant Anna L, Barbosa C, Brum S (2017) Attention to oral health of autistic patient. Rev Pro- Univer SUS 8(1): 67-74.

7. Cruz V, Cruz T, Flag M, Gomes D, Silva L, et al. (2017) Conditioning Strategies in the Dental Care of Patients with Autism Spectrum Disorders. Rev Bras Odontol 74(4): 294-249.

8. Amaral COF, Malacrida VH, Videira FCH, Parizi AGS, Oliveira A De, et al. (2011) Autistic patient: methods and strategies of conditioning and adaptation for dental care. Archives of oral research 8(2): 143-151.

9. Moraes A, Sanchez K, Possobon R, Costa junior A (2004) Psychology and Pediatric Dentistry: The Contribution of Functional Behavior Analysis. Psicol Reflection and Criticism 17(1): 75-82.

10. Do T, Souza N, Viegas Sonegheti J, Helena L, De Andrade R, et al. (2017) Dental Care on a Child with Autistic Spectrum Disorder: Case Report. Rev Odontol Univ Cid São Paul 29(2): 191-198.

11. Purnima Hernandez, Zachary Ikkanda (2011). Applied behavior analysis. The Journal of the American Dental Association 142(3): 281-287.

12. Baer DM, Wolf MM, Risley TR (1968) Some current dimensions of applied behavior analysis. J Appl Behav Anal 1(1): 91-77.

13. Myers SM, Johnson CP (2007) American Academy of Pediatrics Council on Children with Disabilities. Management of children with autism spectrum disorders. Pediatrics 120(5): 1162-1182. 